\title{
General and Exact Inbreeding Coefficient of Maize Synthetics Derived from Three-Way Line Hybrids
}

\author{
Alejandro Ibarra-Sánchez, Juan Enrique Rodríguez-Pérez, Aureliano Peña-Lomelí, \\ Clemente Villanueva-Verduzco and Jaime Sahagún-Castellanos
}

Crop Science Department, Chapingo Autonomus University, Chapingo, 56230, México

*Corresponding Author: Jaime Sahagún-Castellanos. Email: jsahagunc@yahoo.com.mx

Received: 09 February 2021 Accepted: 31 May 2021

\begin{abstract}
Synthetic varieties (SVs) are populations generated by randomly mating their parents. They are a good alternative for low-input farmers who grow onions, maize, and other allogamous crops since the seed produced by a SV does not change from one generation to the next. Although SV progenitors are commonly pure lines, in this case a synthetic $\left(S y n_{T C}\right)$ whose parents are $t$ three-way line crosses, a very common type of maize hybrid grown in Mexico, is studied. The aim was to develop a general and exact equation for the inbreeding coefficient of a $S y n_{T C}$ $\left(F_{m}^{f} S y n_{T C}\right)$ because of its relationship with the mean of economically important traits. This objective arose due to the need for a more advanced study in terms of determining whether $F_{m}^{f} S y n_{T C}$ can be applied specifically and accurately for any number of parents $(t)$, plants per parent $(m)$ and inbreeding coefficient (IC) of the initial lines $\left(F_{L}\right)$. A formula for the IC of the $S y n_{T C}$ was derived that, given any values of $F_{L}\left(0 \leq F_{L} \leq 1\right)$ and $t$, is specific for any value of $m$, not just for "large" numbers associated with the context in which the Hardy-Weinberg law is stated. It was found that $F_{m}^{f} S y n_{T C}$ is very sensitive to changes in $m$ when $m$ is not greater than eight, after which it tends to stabilize very quickly. In summary, unlike previously derived formulas, $F_{m}^{f} S y n_{T C}$ is exact for any values of $t, m$ and $F_{L}$.
\end{abstract}

\section{KEYWORDS}

Genotypic array; genotypic mean; hybrid; random mating; Zea mays L

\section{Introduction}

Synthetic varieties (SVs) of maize (Zea mays L.), also called simply synthetics, are populations generated by randomly mating their parents, usually unrelated inbred lines. In general, they are highly heterozygous and heterogeneous populations that show high adaptability even to adverse environmental conditions such as drought, extreme temperatures, pests, diseases, nutritional problems, etc.

The adaptability of two maize synthetics that had a grain yield of at least $6.0 \mathrm{t} \cdot \mathrm{ha}^{-1} \cdot \mathrm{cycle}^{-1}$ was reported [1]. In a more extensive study on this topic a synthetic variety underwent $S_{1}$ selection over five cycles to improve resistance to Striga hermonthica (Delile) Benth, then selection for grain yield under drought was made. The genetic gain was $423 \mathrm{~kg} \cdot \mathrm{ha}^{-1} \cdot$ cycle $^{-1}$ [2]. A relevant difference between a SV and a hybrid is that the seeds produced by a synthetic must be genetically equal to that of its previous generation. This is not the case in a hybrid. The grain yield of the population producing the harvested 
seeds is often significantly reduced, due to the change in genotypic array that occurs from the hybrid to the following generations. This can strongly influence costs, since in the case of the SV it would not be necessary to buy seeds each production cycle. This cost accounts for up to $20 \%$ of the investment in maize production in Mexico [3].

Synthetic varieties are not only for developing countries, as they also serve, for example, to identify the genotypes or populations whose crosses generate the offspring that have the desired characteristics [4]. Other studies evaluated the prediction efficiency of hybrid maize using diallel analysis and the best linear unbiased predictor (BLUP) [5], methodology described previously [6]. Eight synthetic varieties were sown in a diallel scheme and the hybrids and their parents were then evaluated in three separate environments, with both combining abilities and breeding values (BLUPs) being predicted.

Systematic breeding of synthetic maize varieties is carried out by the International Maize and Wheat Improvement Center (CIMMYT), which breeds open-pollinated varieties for yield potential and tolerance to adverse biotic and abiotic agents. These varieties are synthetics whose parents are the lines that result in the process of developing hybrid varieties [7].

Typically, the parents of a SV are inbred lines. However, the use of single and double crosses as progenitors of this type of variety has been proposed [8]. This modification can reduce costs, labor and time since already existing hybrids can be used to reduce the number of genetic materials that have to be formed and evaluated in field experiments to study the usually numerous SVs that can be formed even with few potential parents.

In particular, the formation of a synthetic variety with $t$ three-way line crosses may be favored by the greater ease of predicting its yield based on the experimental evaluation of $t$ ' potential parents and the $t^{\prime}$ $\left(t^{\prime}-1\right)$ crosses between them [9]. The sum of these numbers $\left[\left(t^{\prime}\right)^{2}\right]$ is less than what would be generated if the potential parents were the $3 t^{\prime}$ lines with which the $t^{\prime}$ three-way line hybrids are formed. It is assumed that $t^{\prime}>t$. Maize grain yield and other variables of economic importance, on the other hand, are inversely related to the inbreeding coefficient (IC) [10]. For a panmictic population of diploid individuals, such as maize, this coefficient is the probability that a random individual has a genotype formed by two identical by descent genes (they are two genes both derived from the same gene in some ancestor). These two genes can be visualized as a random sample of two genes taken with replacement of the set formed by all the genes of the same locus in all the individuals of the population [11].

Maize synthetics formed with three-way line cross hybrids $\left(S y n_{T C}\right)$ are interesting because among hybrid types, three-way line ones are very often cultivated in Mexico and because the gene frequencies of the parent lines of each three-way line cross hybrid (TC) are not equal. On the other hand, there is a gap regarding the exact relationship between the true inbreeding coefficient of this synthetic $\left(F S y n_{T C}\right)$ and the number of plants representing each parent $(m)$. Except in one case where it apparently appears by mistake [8], this number $(m)$ is not included in the most recently developed formulas [12]. In this regard, it is herein hypothesized that because the inbreeding coefficients of an individual generated by self-fertilization and another generated by crossbreeding differ, and because, in the formation of the $S y n_{T C}$, the random mating between the $m$ plants representing each parent, the number of self-fertilizations $(m)$ and that of intraparental crosses $[m(m-1)]$ are not linearly related, $F S y n_{T C}$ must include $m$ in its equation.

It is certainly common to do research on inbreeding, coancestry, etc. in which a "large" population size and absence of migration, mutation and natural selection are implicitly or explicitly assumed. Of these assumptions, some are completely beyond the researcher's control; however, population size is usually determined in some way by the breeder. Desirably, breeders should have an objective basis to guide their decisions about the magnitude of $m$. 
This research aims to derive a general and exact equation for the inbreeding coefficient of synthetics whose parents are three-way line $\operatorname{crosses}\left(F_{m}^{f} S y n_{T C}\right)$, general in the sense that it is applicable for any specific values of $t, F_{L}\left(0 \leq F_{L} \leq 1\right)$ and, particularly, $m$.

\section{Materials and Methods}

This study was based on the model of a locus of a diploid species that reproduces by random mating, such as maize (Zea mays L.), onion (Allium cepa L.), etc. It is considered that since a synthetic variety (SV) is generated by a randomly mating of its parents, the resulting population and those of subsequent generations would have the same genotypic array, unless there is pollen contamination from another population [7]. According to this consideration, the inbreeding coefficient (IC) of a SV is the probability that the genotype of a random individual of such a variety is formed by two identical by descent genes. These two genes can be visualized as a random sample taken with replacement of the set formed by all the genes of the population's gametic array [11].

In particular, if the parents are $t$ three-way line hybrids and each parent is represented by $m$ plants, according to the model of a locus, the gametic array of the SV parents $\left(S y n_{T C}\right)$ is formed by $2 m t$ genes whose individual frequency is uniform $[1 / 2 m t]$. Thus, the IC can be expressed as the probability of the occurrence of an event in a finite sample space.

To derive the IC of the $S y n_{T C}$, it was assumed that: 1) the IC of the initial parent lines is $\left.F_{L}\left(0 \leq F_{L} \leq 1\right) ; 2\right)$ these lines are unrelated; 3 ) each three-way line cross is represented by $m$ plants; and 4) the number of threeway line hybrids, or parents, is $t$. Thus, the $S y n_{T C}$ must be the population resulting from the random mating of $m t$ plants. This implies randomly mating plants from any subset of these $m t$ plants. According to this consideration, only self-fertilization and intraparental mating, the only sources of genotypes consisting of two identical by descent genes in the formation of the $S y n_{T C}$, will contribute to the IC of the $S y n_{T C}$.

According to the above scenario, the IC of a SV formed by $t$ three-way line crosses, each represented by $m$ individuals, must be a quotient. The numerator is the sum of products formed by the number of selffertilizations plus that of two types of intraparental crosses, both multiplied by their corresponding average inbreeding coefficient (probability that the two genes of an individual's genotype taken at random from the corresponding set are identical by descent). The denominator is the sum of the number of selffertilizations and the numbers of intra and interparental crosses generated by the random mating of the $t$ parents represented by $t m$ plants.

In a population produced by randomly mating $t$ three-way line crosses, each represented by $m$ plants, the number of resulting self-fertilizations is $t m$, and the number of intraparental crosses is $t m(m-1)$, because each of the $m$ plants representing a parent is directly and reciprocally crossed with each of the remaining $m-1$ plants. Therefore, as each parent is represented by $m$ plants, the number of self-fertilizations plus the number of intraparental crosses is $t m+t m(m-1)=t^{2}$. Finally, the number of interparental crosses is $t(t-1) m^{2}$ and, consequently, the total number of offspring is $(t m)^{2}$.

\section{Results}

We will consider any values of $t, F_{L}$ and $m$ in a $S y n_{T C}$ to derive its IC $\left(F_{m}^{f} S y n_{T C}\right)$. For this, the information in Tab. 1 will be used. This contains the ICs of the offspring of each cross and selffertilization produced by randomly mating plants whose genotypes are the eight that generate, with the same probability, a three-way line cross of the form $\left(A_{1} A_{2} \times B_{1} B_{2}\right) \times C_{1} C_{2}$. Genotypes $A_{1} A_{2}, B_{1} B_{2}$ and $\mathrm{C}_{1} \mathrm{C}_{2}$ correspond to the three parent lines of the three-way line cross. The IC of the lines was assumed to be $F_{L}$. This implies that, for example, if the probability $(\mathrm{P})$ that $\mathrm{A}_{1}$ and $\mathrm{A}_{2}$ are identical by descent (三) is $F_{L}, \mathrm{P}\left(\mathrm{A}_{1} \equiv \mathrm{A}_{2}\right)=F_{L}$. Obviously, as the lines are unrelated, the genes of the genotypes of the offspring produced by the three-way line cross are not identical by descent. Consequently, the self-fertilization of each plant produced by a three-way line cross has an inbreeding coefficient equal to $1 / 2$. Therefore, 
if $\mathrm{N}_{1}(m)$ is the total number of self-fertilizations resulting from the random mating of each parent, the contribution to $F_{m}^{f} S y n_{T C}\left(\mathrm{C}_{1}\right)$ of the $t$ parents must be:

$C_{1}=\frac{N_{1}\left(\frac{1}{2}\right)}{m^{2} t}$

Table 1: Inbreeding coefficient of the offspring produced by randomly mating the eight genotypes that form the genotypic array of the three-way line cross $\left(\mathrm{A}_{1} \mathrm{~A}_{2} \times \mathrm{B}_{1} \mathrm{~B}_{2}\right) \times \mathrm{C}_{1} \mathrm{C}_{2}$. The inbreeding coefficient of the lines is $F_{L}\left(0 \leq F_{L} \leq 1\right)$

\begin{tabular}{lllllllll}
\hline Genotypes & \multicolumn{7}{c}{ Genotypes } \\
\cline { 2 - 9 } & $\mathrm{A}_{1} \mathrm{C}_{1}$ & $\mathrm{~A}_{1} \mathrm{C}_{2}$ & $\mathrm{~A}_{2} \mathrm{C}_{1}$ & $\mathrm{~A}_{2} \mathrm{C}_{2}$ & $\mathrm{~B}_{1} \mathrm{C}_{1}$ & $\mathrm{~B}_{1} \mathrm{C}_{2}$ & $\mathrm{~B}_{2} \mathrm{C}_{1}$ & $\mathrm{~B}_{2} \mathrm{C}_{2}$ \\
\hline $\mathrm{A}_{1} \mathrm{C}_{1}$ & $\frac{2}{4}$ & $\frac{1+F_{L}}{4}$ & $\frac{1+F_{L}}{4}$ & $\frac{2 F_{L}}{4}$ & $\frac{1}{4}$ & $\frac{F_{L}}{4}$ & $\frac{1}{4}$ & $\frac{F_{L}}{4}$ \\
$\mathrm{~A}_{1} \mathrm{C}_{2}$ & $\frac{1+F_{L}}{4}$ & $\frac{2}{4}$ & $\frac{2 F_{L}}{4}$ & $\frac{1+F_{L}}{4}$ & $\frac{F_{L}}{4}$ & $\frac{1}{4}$ & $\frac{F_{L}}{4}$ & $\frac{1}{4}$ \\
$\mathrm{~A}_{2} \mathrm{C}_{1}$ & $\frac{1+F_{L}}{4}$ & $\frac{2 F_{L}}{4}$ & $\frac{2}{4}$ & $\frac{1+F_{L}}{4}$ & $\frac{1}{4}$ & $\frac{F_{L}}{4}$ & $\frac{1}{4}$ & $\frac{F_{L}}{4}$ \\
$\mathrm{~A}_{2} \mathrm{C}_{2}$ & $\frac{2 F_{L}}{4}$ & $\frac{1+F_{L}}{4}$ & $\frac{1+F_{L}}{4}$ & $\frac{2}{4}$ & $\frac{F_{L}}{4}$ & $\frac{1}{4}$ & $\frac{F_{L}}{4}$ & $\frac{1}{4}$ \\
$\mathrm{~B}_{1} \mathrm{C}_{1}$ & $\frac{1}{4}$ & $\frac{F_{L}}{4}$ & $\frac{1}{4}$ & $\frac{F_{L}}{4}$ & $\frac{2}{4}$ & $\frac{1+F_{L}}{4}$ & $\frac{1+F_{L}}{4}$ & $\frac{2 F_{L}}{4}$ \\
$\mathrm{~B}_{1} \mathrm{C}_{2}$ & $\frac{F_{L}}{4}$ & $\frac{1}{4}$ & $\frac{F_{L}}{4}$ & $\frac{1}{4}$ & $\frac{1+F_{L}}{4}$ & $\frac{2}{4}$ & $\frac{2 F_{L}}{4}$ & $\frac{1+F_{L}}{4}$ \\
$\mathrm{~B}_{2} \mathrm{C}_{1}$ & $\frac{1}{4}$ & $\frac{F_{L}}{4}$ & $\frac{1}{4}$ & $\frac{F_{L}}{4}$ & $\frac{1+F_{L}}{4}$ & $\frac{2 F_{L}}{4}$ & $\frac{2}{4}$ & $\frac{1+F_{L}}{4}$ \\
$\mathrm{~B}_{2} \mathrm{C}_{2}$ & $\frac{F_{L}}{4}$ & $\frac{1}{4}$ & $\frac{F_{L}}{4}$ & $\frac{1}{4}$ & $\frac{2 F_{L}}{4}$ & $\frac{1+F_{L}}{4}$ & $\frac{1+F_{L}}{4}$ & $\frac{2}{4}$ \\
\hline
\end{tabular}

The 56 intraparental crosses in Tab. 1 (not including the 8 self-fertilizations) have an average probability of generating genotypes with identical by descent genes equal to $\left(2+3 F_{L}\right) / 14$. This is the coancestry between different individuals of the genotypic array of a three-way line cross. These 56 intraparental crosses involved in this coancestry, however, are not the only ones if $m>8$. The total number of intraparental crosses like those in Tab. 1 of each parent depends on $m$ (Fig. 1); as $m$ grows and approaches a multiple of 8 , the rate at which the number of intraparental crosses of the type in Tab. 1 increases. This is because the "sides" of the new corresponding tables are getting bigger. However, when $m$ goes from 8 to 9 , from 16 to 17, from 24 to 25 , etc., the number of intraparental crosses considered does not change (Fig. 1). This is because when $m$ $=9,17,25, \ldots$, according to this visualization of the tables, a Tab. 1-type intraparental cross is not expected to occur, but a self-fertilization and 16,32, 48, .. intraparental crosses of a second type, respectively. The difference between the two types of intraparental crosses may be more evident if we consider that those in Tab. 1-type tables form tables that have the structure of a full $8 \times 8$ diallel. Intraparental crosses of the second type do not correspond to a diallel because, according to this visualization, they are in tables that do not include self-fertilizations. 


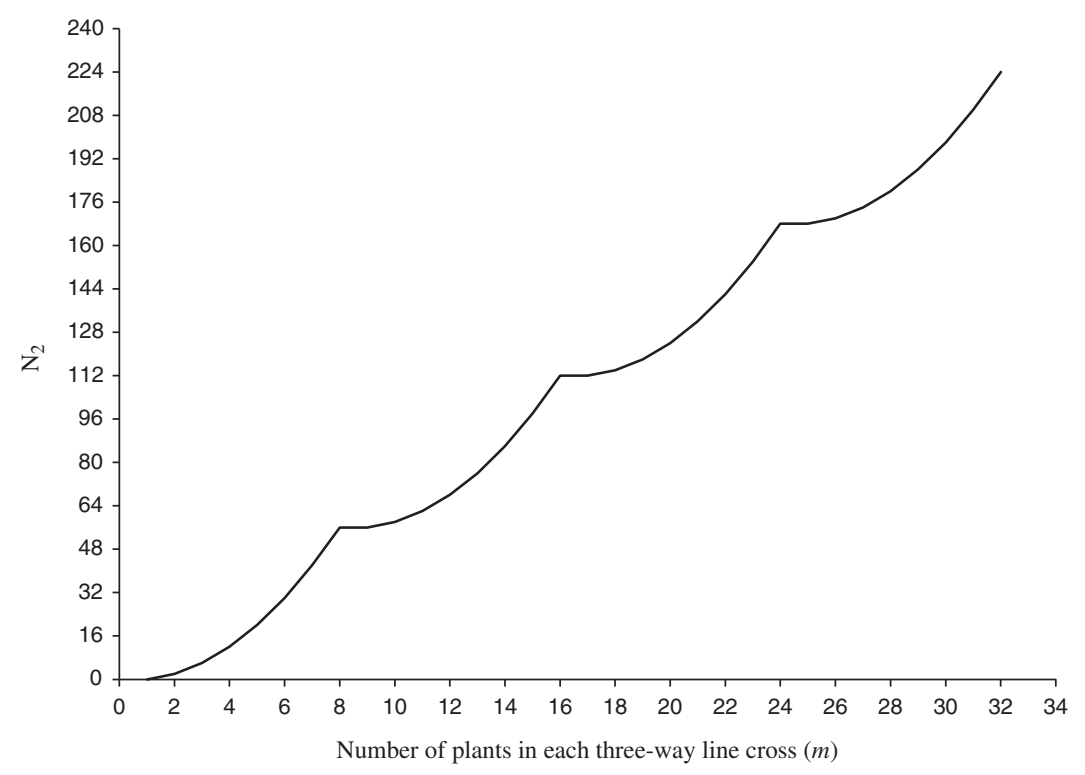

Figure 1: Relationship between the number of plants in each three-way line cross $(m)$ and the number $\left(\mathrm{N}_{2}\right)$ of intraparental crosses (without selfpollinations) among the 8 plants of each complete set of the 8 genotypes forming the genotypic array of a hybrid. If $m / 8$ is not a whole number, the additional corresponding intraparental crosses must be considered in the same way

To determine the contribution of the Tab. 1-type intraparental crosses to $F_{m}^{f} S y n_{T C}$ when $m$ is not a multiple of eight, the existence of the two types of such crosses will be considered because their average inbreeding coefficients differ. The first type is formed by all the direct and reciprocal crosses exemplified in Tab. 1, not including self-fertilizations. Clearly, the number of progenies of this type in each repetition of Tab. 1 is 56. If $\left\lfloor\frac{m}{8}\right\rfloor$ is the number resulting from rounding down $m / 8$ to the nearest whole number, regardless of the resulting decimals, the number of direct and reciprocal intraparental crosses, not including self-fertilizations, in Tab. 1-type tables of each parent is $\left\lfloor\frac{m}{8}\right\rfloor 56$. If $m$ is not a multiple of 8 , the remaining Tab. 1-type intraparental crosses form an incomplete table. The number of intraparental crosses of this incomplete table in each three-way line hybrids is:

$$
\left[\left(\frac{m}{8}-\left\lfloor\frac{m}{8}\right\rfloor\right) 8\right]^{2}-\left(\frac{m}{8}-\left\lfloor\frac{m}{8}\right\rfloor\right) 8
$$

Note that this expression is reduced to zero when $m=9,17,25, \ldots$ Furthermore, it is clear that its second term is the size of one side of the incomplete table (less than eight), which is also equal to the number of terms that form its diagonal. Therefore, the total number of intraparental crosses of the type in Tab. 1 of each parent $\left(\mathrm{N}_{2}\right)$ is:

$N_{2}=\left\lfloor\frac{m}{8}\right\rfloor 56+\left[\left(\frac{m}{8}-\left\lfloor\frac{m}{8}\right\rfloor\right) 8\right]^{2}-\left(\frac{m}{8}-\left\lfloor\frac{m}{8}\right\rfloor\right) 8$

According to the information in Tab. 1, the average inbreeding coefficient of the 56 progenies of intraparental crosses is $\left(2+3 F_{L}\right) / 14$. This is also the average of the 7 intraparental crosses of any row and of any column of the same Tab. 1. With this result and that of Eq. (3), the contribution of the intraparental crosses of the type in Tab. 1 to $F_{m}^{f} \operatorname{Syn}_{T C}\left(\mathrm{C}_{2}\right)$ must be: 
$C_{2}=\frac{\frac{N_{2}\left(2+3 F_{L}\right)}{14}}{m^{2} t}$

The second type of intraparental crosses is partly formed by crosses whose offspring can form complete tables (when $m=16,24,32, \ldots$ ), whose genotypic content is the same as in Tab. 1 . The only difference is that these tables do not have self-fertilizations; instead, they have crosses between two different plants that have the same genotype. It is thus clear that the average inbreeding coefficient of the 64 progenies of each table of this second type is equal to that of Tab. 1 . This average is $3\left(1+F_{L}\right) / 16$. The total number of intraparental progenies of this second type $\left(\mathrm{N}_{3}\right)$ is the sum of those in complete tables $\left(\mathrm{n}_{31}\right)$ plus those in the tables left incomplete when $m$ is not a multiple of $8\left(\mathrm{n}_{32}\right)$. Evidently:

$n_{31}=\left(\left\lfloor\frac{m}{8}\right\rfloor^{2}-\left\lfloor\frac{m}{8}\right\rfloor\right) 64$,

and

$n_{32}=2\left(\frac{m}{8}-\left\lfloor\frac{m}{8}\right\rfloor\right) 8\left\lfloor\frac{m}{8}\right\rfloor 8$

Therefore $N_{3}=n_{31}+n_{32}$.

Since the average inbreeding coefficient in each row or column of a complete table is $3\left(1+F_{L}\right) / 16$, the average inbreeding coefficient of the progenies that form the incomplete tables is also $3\left(1+F_{L}\right) / 16$. Therefore, the average inbreeding coefficient of the offspring of both complete and incomplete tables $\left(F^{f} S y n_{T C}\right)$ for a parent (three-way line cross) is $3\left(1+F_{L}\right) / 16$; that is:

$F^{f} S y n_{T C}=\frac{3\left(1+F_{L}\right)}{16}$

And for $t$ three-way line crosses $\left(F_{t}^{f} S y n_{T C}\right)$ the formula is:

$F_{t}^{f} S y n_{T C}=\frac{3\left(1+F_{L}\right)}{16 t}$

Notably, the total number of intraparental crosses of the second type of one parent $\left(\mathrm{N}_{3}\right)$ and $m$ are nonlinearly related (Fig. 2). For example, according to Eqs. (5) and (6), for $m=1,2,3, \ldots, 8, N_{3}=0$; however, for $m=16,24,32, \ldots, N_{3}=128,384,768, \ldots$, respectively. According to Eqs. (5)-(7), the contribution of these crosses to $F_{m}^{f} \operatorname{Syn}_{T C}\left(\mathrm{C}_{3}\right)$ is:

$C_{3}=\frac{N_{3}\left[\frac{3\left(1+F_{L}\right)}{16}\right]}{m^{2} t}$

In summary, according to Eqs. (1), (4) and (8), the formula for $F_{m}^{f} S y n_{T C}$ is:

$F_{m}^{f} S y n_{T C}=\frac{N_{1}\left(\frac{1}{2}\right)}{m^{2} t}+\frac{\frac{N_{2}\left(2+3 F_{L}\right)}{14}}{m^{2} t}+\frac{N_{3}\left[\frac{3\left(1+F_{L}\right)}{16}\right]}{m^{2} t}$

where: 
$N_{1}=m$
$N_{2}=\left\lfloor\frac{m}{8}\right\rfloor 56+\left\lfloor\left(\frac{m}{8}-\left\lfloor\frac{m}{8}\right\rfloor\right) 8\right]^{2}-\left(\frac{m}{8}-\left\lfloor\frac{m}{8}\right\rfloor\right) 8$
$N_{3}=\left(\left\lfloor\frac{m}{8}\right\rfloor^{2}-\left\lfloor\frac{m}{8}\right\rfloor\right) 64+2\left(\frac{m}{8}-\left\lfloor\frac{m}{8}\right\rfloor\right) 8\left\lfloor\frac{m}{8}\right\rfloor 8$

Note, if $m$ is a multiple of 8 , the number of complete tables $(\mathrm{C})$ is a whole number, and:

$N_{1}=8 C$

$N_{2}=56 C$

$N_{3}=\left(\left\lfloor\frac{m}{8}\right\rfloor^{2}-\left\lfloor\frac{m}{8}\right\rfloor\right) 64=64 C(C-1)$

and

$m=8 C$

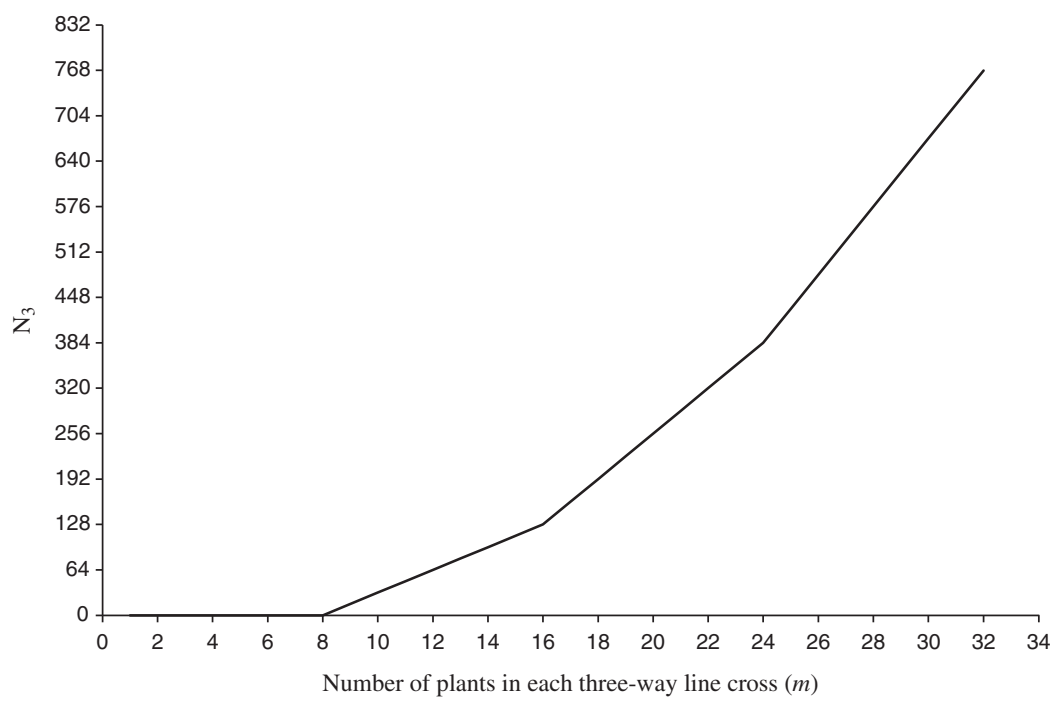

Figure 2: Relationship between the number of plants representing each three-way line hybrid $(m)$ and the number of intraparental crosses $\left(\mathrm{N}_{3}\right)$ each involving 2 plants from different complete or incomplete sets of 8 plants whose genotypes are those forming the genotypic array of a three-way line hybrid

As expected, if $m$ is a multiple of 8, according to Eqs. (10)-(13), $F_{m}^{f} S y n_{T C}$ (Eq. (9)) reduces to the equation for an IC matching that of Eq. (7) derived here for this case. This formula for the IC of the $S y n_{T C}$ was also found in another study [12]. Such a formula, however, does not have the necessary generality to be applied with complete accuracy when $m$ is small or is not a multiple of 8 , although the differences may be small. For example, for $m=8, t=1$ and $F_{L}=1$, the IC is $3\left(1+F_{L}\right) / 16=0.375$, while for $m=9$, it is (Eq. (9)) $[9(1 / 2)+56(5 / 14)+16(6 / 16)] / 81=0.3765$. In addition, if $m<8$ and $0 \leq F_{L} \leq 1$, the inbreeding coefficient of the synthetic thus formed $\left(F_{p}^{f} S y n_{T C}\right)$ must be what results from considering that the contributions to inbreeding come only from self-fertilizations and intraparental crosses that are only of the type shown in Tab. 1. Therefore, what remains is reduced to the equation that results from removing the third term of Eq. (9). The resulting equation is equal to: 
$F_{p}^{f} S y n_{T C}=\frac{1}{2 m t}+\frac{(m-1)\left(2+3 F_{L}\right)}{14 m t}$

Clearly, in this case $\left(m<8\right.$ and $\left.0 \leq F_{L} \leq 1\right), N_{1}=m, N_{2}=m(m-1)$ and $N_{3}=0$. For example, if $F_{L}=1, t=$ 1 and $m=4$ (four self-fertilizations and 12 intraparental crosses of the Tab. 1 -type), the IC is (Eq. (14)) $1 / 8+$ $3 \times 5 / 56=0.3929$.

Eqs. (9) and (14) show that the IC of $S y n_{T C}$ does depend on $m$ and that the relationship between the two is inverse. Additionally, Tab. 2 shows that: 1) the decrease in $F_{m}^{f} S y n_{T C}$ due to increases in $m$ is more intense as $F_{L}$ gets smaller, 2) the largest changes in $F_{m}^{f} S y n_{T C}$ occur in the $m$-value range from 1 to 8 ; and 3) from $m=8$ the differences between the $F_{m}^{f} S y n_{T C}$ values are marginal.

Table 2: Inbreeding coefficient (IC) of synthetic varieties generated with $t$ three-way line hybrids developed with unrelated lines with five ICs $\left(F_{L}\right)$ and represented by 16 plant numbers $(m)$

\begin{tabular}{clllll}
\hline$m$ & \multicolumn{5}{c}{$F_{L}$} \\
\cline { 2 - 6 } & 0.000 & 0.500 & 0.750 & 0.875 & 1.00 \\
\hline 1 & 0.50 & 0.50 & 0.50 & 0.50 & 0.50 \\
2 & 0.32 & 0.38 & 0.40 & 0.42 & 0.43 \\
3 & 0.26 & 0.33 & 0.37 & 0.39 & 0.40 \\
4 & 0.23 & 0.31 & 0.35 & 0.37 & 0.39 \\
5 & 0.21 & 0.30 & 0.34 & 0.36 & 0.39 \\
6 & 0.20 & 0.29 & 0.34 & 0.36 & 0.38 \\
7 & 0.19 & 0.29 & 0.33 & 0.35 & 0.38 \\
8 & 0.19 & 0.28 & 0.33 & 0.35 & 0.38 \\
9 & 0.19 & 0.28 & 0.33 & 0.35 & 0.38 \\
10 & 0.19 & 0.28 & 0.33 & 0.35 & 0.38 \\
11 & 0.19 & 0.28 & 0.33 & 0.35 & 0.38 \\
12 & 0.19 & 0.28 & 0.33 & 0.35 & 0.38 \\
13 & 0.19 & 0.28 & 0.33 & 0.35 & 0.38 \\
14 & 0.19 & 0.28 & 0.33 & 0.35 & 0.38 \\
15 & 0.19 & 0.28 & 0.33 & 0.35 & 0.38 \\
16 & 0.19 & 0.28 & 0.33 & 0.35 & 0.38 \\
$\infty$ & 0.1875 & 0.281 & 0.328 & 0.351 & 0.375 \\
\hline Note: The ICs of the synthetic varieties are obtained by multiplying the values in the table by (1/t).
\end{tabular}

Regarding the methodology used in this research, it is not unique to problems like the one solved here. Another, probably more complex, methodology for the case of a synthetic developed with double crosses has also been proposed [13].

\section{Discussion}

An inbreeding coefficient of a synthetic variety whose parents are $t$ three-way line crosses made with pure lines $\left(F_{L}=1\right)$ was derived [3]. The inbreeding coefficient $\left(F^{M} S y n_{T C}\right)$ obtained is $(3 m+1) /(8 t m)=3 /$ $8 t+1 / 8 \mathrm{tm}$. If the conditions for which this author derived $F^{M} \operatorname{Syn}_{T C}\left(F_{L}=1\right.$ and $m$ is a multiple of 8$)$ are 
applied to $F_{t}^{f} S y n_{T C}$ (Eq. (7)), then based on Eqs. (9), (11), and (13) for this case:

$$
\begin{aligned}
F_{t}^{f} \operatorname{Syn}_{T C} & =\frac{m\left(\frac{1}{2}\right)}{m^{2} t}+\frac{56 C\left(2+3 F_{L}\right)}{14 m^{2} t}+\frac{64 C(C-1) 3\left(1+F_{L}\right)}{16 m^{2} t} \\
& =\frac{1}{2 m t}+\frac{56\left(2+3 F_{L}\right)}{(8 m t)(14)}+\frac{8(C-1) 3\left(1+F_{L}\right)}{16 m t} \\
& =\frac{1}{2 m t}+\frac{2+3 F_{L}}{2 m t}+\frac{(m-8) 3\left(1+F_{L}\right)}{16 m t} \\
& =\frac{1}{2 m t}+\frac{5}{2 m t}+\frac{6(m-8)}{16 m t} \\
& =\frac{3 m}{8 m t} \\
& =\frac{3}{8 t}
\end{aligned}
$$

This means that, effectively, $F^{M} S y n_{T C}$ has a bias equal to $1 /(8 \mathrm{tm})$ [3]. This bias is due to the fact that this inbreeding coefficient was derived using the formula:

$F^{M} S y n_{T C}=\left[\frac{1}{2 m t}\right]\left[1+2 m(t-1) r_{O, B}+2(m-1) r_{O, W}^{\prime}+F_{O}\right]$

where:

$r_{O, W}^{\prime}=$ coancestry between plants representing a three-way line hybrid

$F_{O}=$ parents' inbreeding coefficient

$r_{O, B}=$ coancestry between individuals of different hybrids

Because the lines are unrelated, $F_{O}$ and $r_{O, B}$ were correctly considered equal to zero. In a previous study [3], however, it was considered that if $F=1, r_{O, W}^{\prime}=3 / 8$. According to the derivation made in a more recent study [12], the coancestry between individuals representing each parent $\left(r_{O, W}\right)$ is:

$r_{O, W}=\frac{3 m\left(1+F_{L}\right)-8}{16(m-1)}$

This formula, accordingly, does not include the coancestry of an individual with itself that is the IC of the offspring generated by self-fertilization. In a previous study [3] it was included, although it was already included in Eq. (15) in the form $\left(1+F_{O}\right) /(2 m t)$. According to Eq. (16), if $F_{L}=1, r_{O, W}$ reduces to the form:

$r_{O, W}=\frac{3 m-4}{8(m-1)}=\frac{3}{8}-\frac{1}{8(m-1)}$

This implies that $r_{O, W}^{\prime}$ has a bias equal to $\frac{1}{[8(m-1)]}$.

According to the data in Tab. 1, the inbreeding coefficient of the progenies of the intraparental crosses of the first type, which is equal to the coancestry between the genotypes that form the genotypic array of a threeway line cross $\left(r_{O, W}\right)$, is:

$r_{O, W}=\frac{2+3 F_{L}}{14}$ 
If in addition $F_{L}=1$, this coancetry reduces to $5 / 14$. This result also corresponds to that of Eq. (17) when $m=8$.

The IC of the $S y n_{T C}$, however, is expected to be higher than the IC of the synthetic whose parents are the $3 t$ parent lines of that $S y n_{T C}$. This is because by randomly mating the parents the maximum frequency of genotypes that are not formed by identical by descent genes is only generated when the frequencies of the genes are the same. This does not occur in the formation of $S y n_{T C}$ because the gene frequencies of the three lines of each three-way line hybrid are not uniform. The frequency of the genes contributed by each of the two lines that form the single cross is half of that contributed by the third line. The size of $m$ is also important in this context. Gene frequencies should tend to stabilize as $m$ is larger and consequently the IC as well. The opposite should happen as $m$ is smaller. The interesting aspect is to determine when stabilization starts.

\section{Conclusions}

An equation was derived for the inbreeding coefficient of a synthetic developed with three-way line maize hybrids $\left(F_{m}^{f} S y n_{T C}\right)$. Unlike the equations previously proposed for the specific case in which the initial parent lines are pure, it has the flexibility to quantify the general and exact inbreeding coefficient of the synthetic whose parents are $t$ three-way line hybrids. $F_{m}^{f} S y n_{T C}$ is general because it is applicable regardless of the number of parents, the level of inbreeding of the lines and, particularly, the number of plants representing each three-way line cross $(m)$. And it is exact because it has no biases or inaccuracies due to small $m$ values and errors of interpretation. Furthermore, $F_{m}^{f} S y n_{T C}$ does include the number of plants per parent $(m)$. This number has its greatest effects when it is less than eight and its relationship with $F_{m}^{f} S y n_{T C}$ is inverse. For values of $m$ greater than eight, the differences between the inbreeding coefficients are marginal.

Authors' Contributions: Conceptualization: Alejandro Ibarra-Sánchez and Juan Enrique Rodríguez-Pérez Data curation: Juan Enrique Rodríguez-Pérez and Jaime Sahagún-Castellanos. Formal analysis: Jaime Sahagún-Castellanos. Funding acquisition: Aureliano Peña-Lomelí. Investigation: Alejandro IbarraSánchez. Methodology: Alejandro Ibarra-Sánchez. Project administration: Clemente Villanueva-Verduzco. Resources: Aureliano Peña-Lomelí. Supervision: Aureliano Peña-Lomelí, Clemente Villanueva-Verduzco and Jaime Sahagún-Castellanos. Validation: Aureliano Peña-Lomelí. Writing original draft: Alejandro Ibarra-Sánchez and Clemente Villanueva-Verduzco. Writing review and editing: Juan Enrique RodríguezPérez, Aureliano Peña-Lomelí and Jaime Sahagún-Castellanos.

Funding Statement: The authors received no specific funding for this study.

Conflicts of Interest: The authors declare that they have no conflicts of interest related to report regarding the present study.

\section{References}

1. Farid, M., Musa, Y., Nasarudolin, R. I. (2019). Selection of various synthetic maize (Zea mays L.) genotypes on drought stress condition. Earth and Environmental Science, 235, 1-6. DOI 10.1088/1755-1315/235/1/012027.

2. Badu-Apraku, B., Ilfie, B., Talabi, A., Obeng-Bio, E., Asiedu, R. (2018). Genetic variances and heritabilities of traits of an early yellow maize population after cycles of improvement for striga resistance and drought tolerance. Crop Science, 58, 2261-2273. DOI 10.2135/cropsci2017.10.0628.

3. Márquez-Sánchez, F. (2010). Inbreeding coefficient and mean prediction of maize of three-way lines hybrids. Maydica, 55, 227-229.

4. Saboor, A., Ulla, H., Shahurar, D., Fahad, S., Khan, N. et al. (2018). Heritability and correlation analysis of morphological and yield traits in maize. Journal of Plant Biology Crop Research, 2, 1-8. DOI 10.33582/2637-7721. 
5. Oliveira, G., Buzinaro, R., Revolti, L., Giorgenon, C., Charnai, K. et al. (2016). An accurate prediction of maize crosses using diallel analysis and best linear unbiased predictor (BLUP). Chilean Journal of Agricultural Research, 16, 294-297. DOI 10.4067/S0718-58392016000300005.

6. Bernardo, R. (2010). Breeding for quantitative traits in plants, vol 1, pp. 369. Woodbury, USA: Stemma Press.

7. Masuka, B., Magorokosho, C., Olsen, M., Atlin, G., Bänziger, M. et al. (2017). Gains in maize genetic improvement in Eastern and Southern Africa II. CIMMYT open-pollinated variety, breeding pipeline. Crop Science, 57, 180-191. DOI 10.2135/cropsci2016.05.0408.

8. Márquez-Sánchez, F. (2011). Maize synthetics from a mixture of single, three-way, and double cross hybrids using inbreeding coefficients and mean prediction. Maydica, 56, 341-342.

9. Wright, S. (1922). The effects of inbreeding and crossbreeding on guinea pigs. no. 1090. USA: US Government Printing Office.

10. Busbice, T. (1970). Predicting yield of synthetic varieties. Crop Science, 32, 271-274. DOI 10.2135/ cropsci1970.0011183X001000030017x.

11. Sahagún-Castellanos, J., Rodríguez-Peréz, J. E., Escalante-González, J. L. (2013). Yield prediction and inbreeding of maize synthetics generated with lines and single crosses. Classic Probability. Revista de la Facultad de Ciencias Agrarias, 45(2), 75-84.

12. Ibarra-Sánchez, A., Rodríguez-Pérez, J. E., Sahagún-Castellanos, J. (2019). General inbreeding coefficient of maize synthetics derived from three-way line hybrids. Terra Latinoamericana, 37, 479-485. DOI 10.28940/ terra.v37i4.545.

13. Rodríguez-Pérez, J., Peña-Lomelí, A., Villanueva-Verduzco, C., Sahagún-Castellanos, J. (2019). General and exact inbreeding coefficient of maize synthetics derived from double crosses. Agrociencia, 53, $235-244$. 